\title{
Fundamental principles of structural behaviour under thermal effects
}

\author{
A.S. Usmani*, J.M. Rotter, S. Lamont, A.M. Sanad, M. Gillie \\ School of Civil and Environmental Engineering, University of Edinburgh, Crew Building, \\ The King's Buildings, Edinburgh EH9 $3 J N, U K$
}

Received 10 July 2000; received in revised form 15 December 2000; accepted 22 March 2001

\begin{abstract}
This paper presents theoretical descriptions of the key phenomena that govern the behaviour of composite framed structures in fire. These descriptions have been developed in parallel with large scale computational work undertaken as a part of a research project (The DETR-PIT Project, Behaviour of steel framed structures under fire conditions) to model the full-scale fire tests on a composite steel framed structure at Cardington (UK). Behaviour of composite structures in fire has long been understood to be dominated by the effects of strength loss caused by thermal degradation, and that large deflections and runaway resulting from the action of imposed loading on a 'weakened' structure. Thus 'strength' and 'loads' are quite generally believed to be the key factors determining structural response (fundamentally no different from ambient behaviour). The new understanding produced from the aforementioned project is that, composite framed structures of the type tested at Cardington possess enormous reserves of strength through adopting large displacement configurations. Furthermore, it is the thermally induced forces and displacements, and not material degradation that govern the structural response in fire. Degradation (such as steel yielding and buckling) can even be helpful in developing the large displacement load carrying modes safely. This, of course, is only true until just before failure when material degradation and loads begin to dominate the behaviour once again. However, because no clear failures of composite structures such as the Cardington frame have been seen, it is not clear how far these structures are from failure in a given fire. This paper attempts to lay down some of the most important and fundamental principles that govern the behaviour of composite frame structures in fire in a simple and comprehensible manner. This is based upon the analysis of the response of single
\end{abstract}

*Corresponding author. Tel.: + 44-131-650-5789; fax: + 44-131-650-6781.

E-mail address: asif.usmani@ed.ac.uk (A.S. Usmani). 
structural elements under a combination of thermal actions and end restraints representing the surrounding structure. (C) 2001 Elsevier Science Ltd. All rights reserved.

Keywords: Composite structures; Thermal expansion; Thermal bowing; Restraint to thermal actions; Non-linear geometrical responses

\section{Introduction}

This paper is based upon work undertaken as a part of a large multi-organisation project of modelling the behaviour of steel framed structures in fire [1] (namely the full-scale tests at Cardington [2]). In executing this project and identifying the key governing phenomena it was found necessary to make use of the fundamental principles repeatedly in order to understand the complex interactions of the different structural mechanisms taking place. This led to the development of a number of important principles that were found to govern the overall behaviour of the structure. These principles are very useful in interpreting the results from much larger and sophisticated computational models and in helping to develop a coherent picture of the behaviour. Most of these ideas have already been presented at the INTERFLAM [3] and $\mathrm{SiF}[4]$ conferences.

This work was undertaken because the assessment of the adequacy of composite steel frame structures in fire continues to be based upon the performance of isolated elements in standard furnace tests. This is despite the widespread acceptance amongst structural engineers that such an approach is over-conservative and even more importantly unscientific. This view has gained considerable strength in the aftermath of the Broadgate fire [5] and has been reinforced by the Cardington tests. Current codes such as BS 5950 Part 8 and EC3 (draft) allow designers to take advantage of the most recent developments in the field by treating fire-related loading as another limit state. The advances in understanding structural behaviour in fire achieved in the last few years have been considerable with a large number of groups across Europe undertaking extensive research projects and concentrating on a number of different aspects of structural behaviour in fire [6-9]. These advances combined with the findings of the DETR-PIT project [1] make it possible for engineers to treat the design for fire in an integrated manner with the design of a structure for all other types of loading. This can be done by using the numerical modelling tools that have been instrumental in developing this understanding. However, the use of such tools, which are indispensable for research, is not practical in the design office. Exploitation of the new knowledge can only become feasible in practice if the understanding generated is further developed into simpler analytical expressions, enabling consulting engineers and designers to undertake performancebased design of steel frame structures without having to resort to large scale computation. The principles presented here constitute a step towards generating the analytical tools necessary for such use.

All analytical expressions developed in this paper have been developed ab initio from fundamental structural mechanics. The most fundamental relationship that 
governs the behaviour of structures when subjected to thermal effects is

$$
\varepsilon_{\text {total }}=\varepsilon_{\text {thermal }}+\varepsilon_{\text {mechanical }}
$$

with

$$
\varepsilon_{\text {mechanical }} \rightarrow \sigma \quad \text { and } \quad \varepsilon_{\text {total }} \rightarrow \delta
$$

The total strains govern the deformed shape of the structure $\delta$ through kinematic or compatibility considerations. By contrast, the stress state in the structure $\sigma$ (elastic or plastic) depends only on the mechanical strains. Where the thermal strains are free to develop in an unrestricted manner, there are no external loads, axial expansion or thermal bowing results from

$$
\varepsilon_{\text {total }}=\varepsilon_{\text {thermal }} \quad \text { and } \quad \varepsilon_{\text {total }} \rightarrow \delta .
$$

By contrast, where the thermal strains are fully restrained without external loads, thermal stresses and plastification result from

$$
0=\varepsilon_{\text {thermal }}+\varepsilon_{\text {mechanical }} \quad \text { with } \quad \varepsilon_{\text {mechanical }} \rightarrow \sigma .
$$

The single most important factor that determines a real structure response to heating is the manner in which it responds to the unavoidable thermal strains induced in its members through heating. These strains take the form of thermal expansion to an increased length (under an average centroidal temperature rise) and curvature (induced by a temperature gradient through the section depth). If the structure has an insufficient end translational restraint to thermal expansion, the considerable strains are taken up in expansive displacements, producing a displacementdominated response. Thermal gradients induce curvature leading to bowing of a member whose ends are free to rotate, again producing large displacements (deflections).

Members whose ends are restrained against translation produce opposing mechanical strains to thermal expansion strains and therefore large compressive stresses (see Eq. (1)). Curvature strains induced by the thermal gradient in members whose ends are rotationally restrained can lead to large hogging (negative) bending moments throughout the length of the member without deflection. The effect of induced curvature in members whose ends are rotationally unrestrained, but translationally restrained, is to produce tension.

Therefore, for the same deflection in a structural member, a large variety of stress states can exist, large compressions where restrained thermal expansion is dominant and very low stresses where the expansion and bowing effects balance each other; in the cases where thermal bowing dominates, tension occurs in laterally restrained and rotationally unrestrained members, while large hogging moments occur in rotationally restrained members. The variety of responses can indeed exist in real structures if one imagines the different types of fire a structure may be subjected to. A fast burning fire that reaches flashover and high temperatures quickly and then dies off can produce high thermal gradients (hot steel and relatively cold concrete) but lower mean temperatures. By contrast, a slow fire that reaches only modest temperatures but burns for a long time could produce considerably higher mean temperature and lower thermal gradients. 
Most situations in real structures under fire have a complex mix of mechanical strains due to applied loading and mechanical strains due to restrained thermal expansion. These lead to combined mechanical strains which often far exceed the yield values, resulting in extensive plastification. The deflections of the structure, by contrast, depend only on the total strains, so these may be quite small where high restraint exists, but they are associated with extensive plastic straining. Alternatively, where less restraint exists, larger deflections may develop, but with a lesser demand for plastic straining and hence a lesser destruction of the stiffness properties of the materials. These relationships, which indicate that larger deflections may reduce material damage and correspond to higher stiffnesses, or that restraint may lead to smaller deflections with lower stiffnesses, can produce structural situations which appear to be quite counter-intuitive if viewed from a conventional (ambient) structural engineering perspective.

The ideas presented above will be more formally explored in the following sections in the context of simple structural configurations and analytical expressions will be developed for many cases of fundamental importance.

\section{Thermal expansion}

Heating induces thermal expansion strains (say $\varepsilon_{\mathrm{T}}$ ) in most structural materials. These are given by

$$
\varepsilon_{\mathrm{T}}=\alpha \Delta T \text {. }
$$

If a uniform temperature rise, $\Delta T$, is applied to a simply supported beam without axial restraint, the result will simply be an expansion or increase in length of $l \alpha \Delta T$ as shown in Fig. 1. Therefore the total strain ( $\operatorname{say} \varepsilon_{t}$ ) is equal to the thermal strain and there is no mechanical strain (say $\varepsilon_{\mathrm{m}}$ ) which means that no stresses develop in the beam.

\subsection{Thermal expansion against rigid lateral restraints}

Clearly, beams in real structure do not have the freedom to elongate in the manner described above. Therefore, a more realistic case is to consider an axially restrained beam subjected to a uniform temperature rise, $\Delta T$ (as shown in Fig. 2). It is clear to see that in this case the total strain $\varepsilon_{\mathrm{t}}$ is zero (no displacements). This is because the

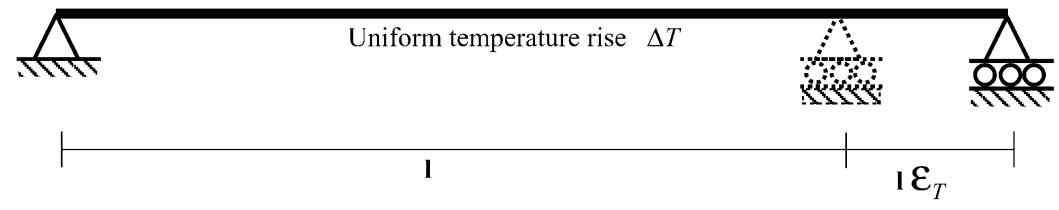

Fig. 1. Uniform heating of a simply supported beam. 


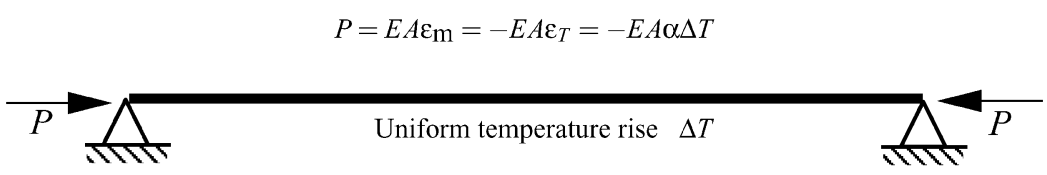

Fig. 2. Axially restrained beam subjected to uniform heating.

thermal expansion is cancelled out by equal and opposite contraction caused by the restraining force $P$ (i.e. $\varepsilon_{\mathrm{t}}=\varepsilon_{\mathrm{T}}+\varepsilon_{\mathrm{m}}=0$ therefore $\varepsilon_{\mathrm{T}}=-\varepsilon_{\mathrm{m}}$ ). There now exists a uniform axial stress $\sigma$ in the beam equal to $E \varepsilon_{\mathrm{m}}$. The magnitude of the restraining force $P$ is,

$$
P=E A \varepsilon_{\mathrm{m}}=-E A \varepsilon_{\mathrm{T}}=-E A \alpha \Delta T .
$$

If the temperature is allowed to rise indefinitely, then there will be two basic responses, depending upon the slenderness of the beam:

1. If the beam is sufficiently stocky, then the axial stress will sooner or later reach the yield stress $\sigma_{\mathrm{y}}$ of the material and if the material has an elastic-plastic stress-strain relationship, then the beam will continue to yield without any further increase in stress, but it will also store an increasing magnitude of plastic strains. The yield temperature increment $\Delta T_{\mathrm{y}}$ is,

$$
\Delta T_{\mathrm{y}}=\frac{\sigma_{\mathrm{y}}}{E \alpha} .
$$

2. If the beam is slender then it will buckle before the material reaches its yield stress. The Euler buckling load $P_{\text {cr }}$ for a beam/column, as in Fig. 2 is

$$
P_{\mathrm{cr}}=\frac{\pi^{2} E I}{l^{2}} \text {. }
$$

Equating this to the restraining force $P$, we have,

$$
E A \alpha \Delta T=\frac{\pi^{2} E I}{l^{2}}
$$

which leads to a critical buckling temperature of,

$$
\Delta T_{\mathrm{cr}}=\frac{\pi^{2}}{\alpha}\left(\frac{r}{l}\right)^{2}
$$

or

$$
\Delta T_{\mathrm{cr}}=\frac{\pi^{2}}{\alpha \lambda^{2}},
$$

where $r$ is the radius of gyration and $\lambda$ is the slenderness ratio $(l / r)$. This expression is valid for other end-restraint conditions if $l$ is interpreted as the effective length.

In this case, if the temperature is allowed to rise further, then the total restraining force will stay constant (assuming an elastic material and no thermal 
degradation of properties) and the thermal expansion strains will continue to be accommodated by the outward deflection of the beam $\delta$ as shown in Fig. 3.

The above cases represent the two fundamental responses in beams subjected to restrained thermal expansion. Either of the two (yielding or buckling) may occur on its own (based upon the slenderness of the beam) or a more complex response consisting of a combination of yielding and buckling may also occur.

The pattern of the development of deflections, axial compression forces and moments with an increase in temperature in slender restrained elastic beams is as shown in Figs. 4 and 5 (it is the patterns that are important to note here and not the

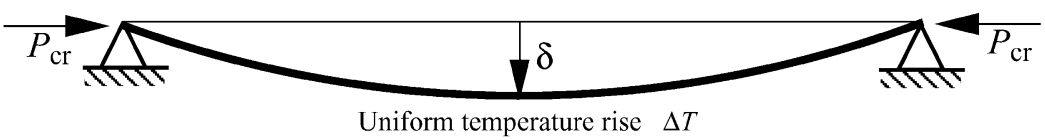

Fig. 3. Buckling of an axially restrained beam subjected to uniform heating.
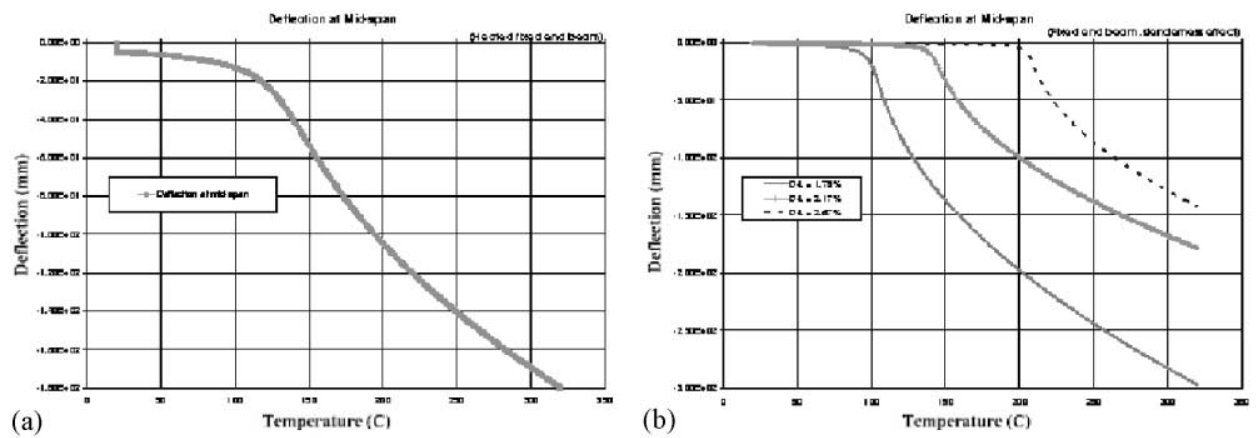

Fig. 4. Deflection of axially restrained elastic beams subjected to heating: (a) Single beam, (b) three beams of varying slenderness.
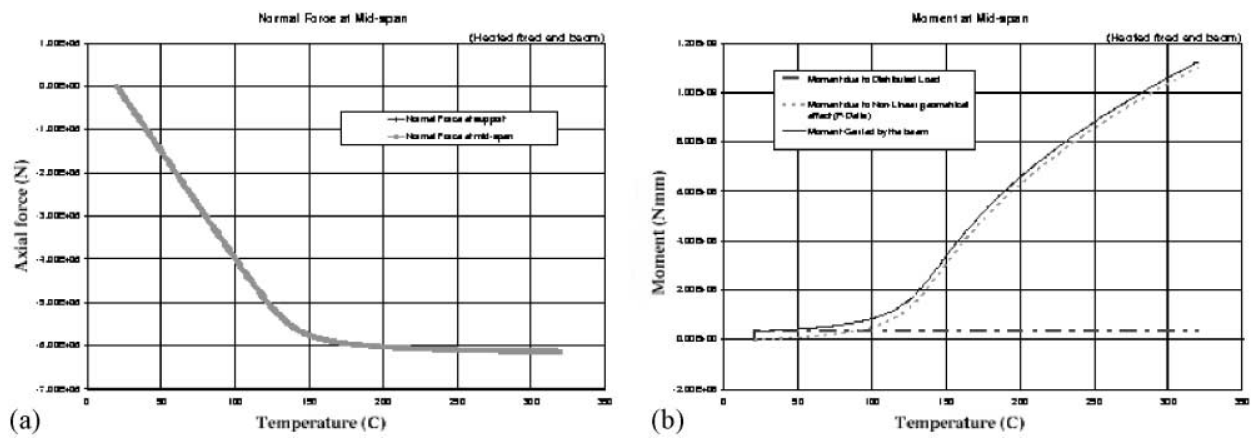

Fig. 5. Forces in an axially restrained elastic beam subjected to heating: (a) axial forces, and (b) moments. 
actual values, these figures are explained in more detail in [3]). The deflection and axial force figures clearly show a pre-buckling and post-buckling type response. The sharp bifurcation pattern is absent as a uniformly distributed load is imposed on the beam, imparting an initial displacement to it. The midspan moment continues to rise even after buckling as it consists mainly of the $P-\delta$ moment generated by the axial restraint force times of the midspan deflection (which continues to rise beyond buckling).

Ideal elastic properties were assumed when discussing the case of buckling above. If the properties are ideal elasto-plastic, then the deflections and axial compression variations will have a pattern as shown in Fig. 6 (again it is the patterns that are important to note). If the properties remain elastic, albeit with a uniform degradation with temperature, the pattern of deflection and axial compression in the beam changes to the one shown in Fig. 7. Clearly, the response of real composite beams subject to restrained thermal expansion will consist of a combination of the responses shown here. That this is indeed the case, can be seen in [10], where the results of modelling the British steel restrained beam test are shown (which comes
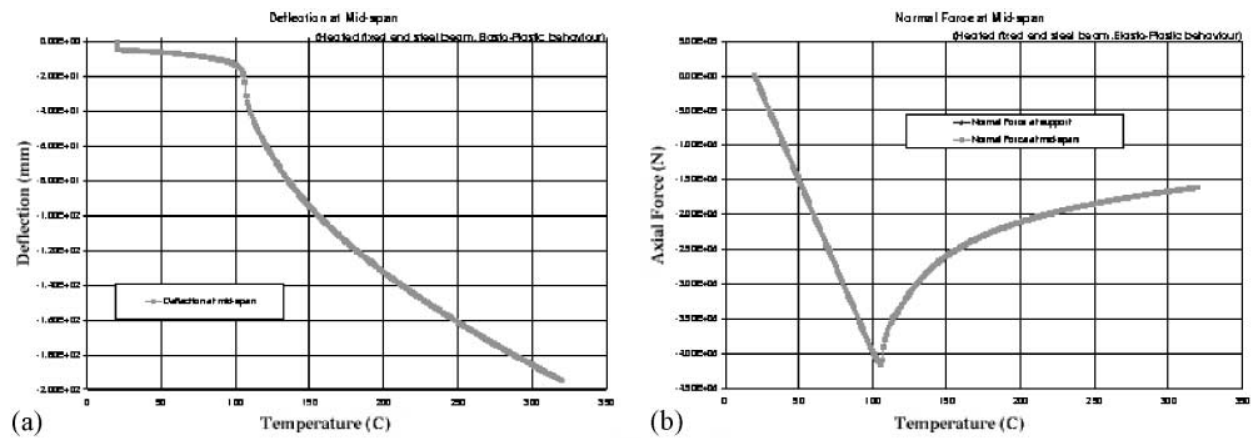

Fig. 6. Deflections (a) and axial forces (b), in an axially restrained elastic-plastic beam.
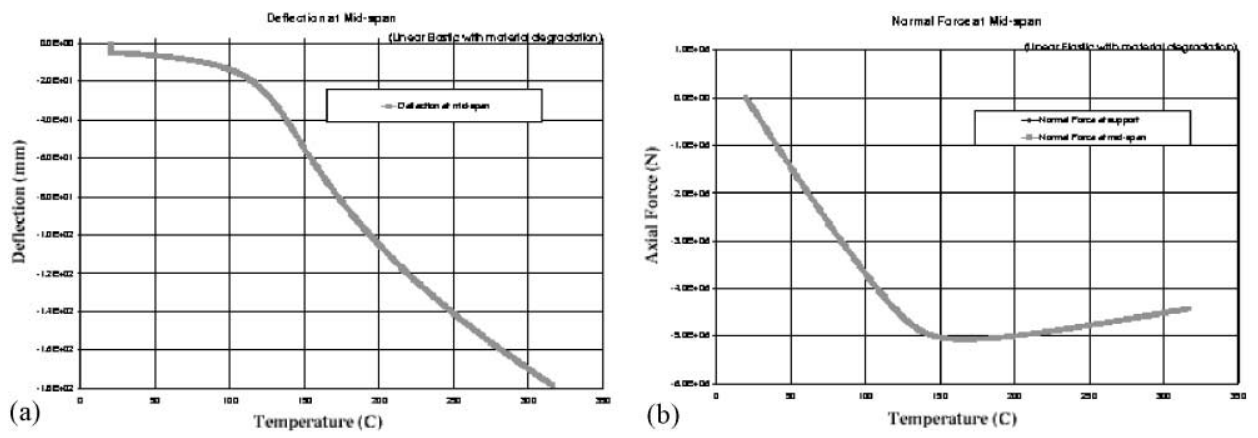

Fig. 7. Deflections (a) and axial forces (b), in a restrained beam with reducing elastic stiffness. 
closest to the ideal case of rigid lateral restraint). There are other factors in that test that govern the response of the heated composite beam, particularly the effect of deflection compatibility in the two directions; however, the similarity of the development of axial forces in the steel joist and the composite beam to the patterns shown here is clear to see. Therefore, reduction in stiffness by heating reduces the magnitude of the axial compression force in the restrained beam, and when the beam material reaches its yield value, a much more rapid reduction in the restraining force occurs with a corresponding rise in deflections.

\subsection{Thermal expansion against finite lateral restraints}

In the previous discussion we have assumed the axial restraints to be perfectly rigid. This is an upper limit and practically impossible to achieve in real structures which offer only finite restraints. Fig. 8 shows such a beam restrained axially by a translational spring of stiffness $k_{\mathrm{t}}$. The compressive axial stress developed by thermal expansion is

$$
\sigma=\frac{E \alpha \Delta T}{\left(1+E A / k_{\mathrm{t}} L\right)}
$$

and critical buckling temperature is now given by

$$
\Delta T_{\mathrm{cr}}=\frac{\pi^{2}}{\alpha \lambda^{2}}\left(1+\frac{E A}{k_{\mathrm{t}} L}\right)
$$

From Eq. (8) it can be seen that buckling and post-buckling phenomena should be observable at moderate fire temperatures in structures with translational restraint stiffnesses $\left(k_{\mathrm{t}}\right)$ which are quite comparable with the axial stiffness of the member $(E A / L)$. Fig. 9 shows a plot derived from Eq. (8), where critical buckling temperatures are plotted against slenderness ratio for different restraint stiffnesses. The results clearly show that the amount of restraint required is not large for slender sections to reach buckling temperature. Bearing in mind that the axial stiffness of the member $(E A / L)$ is reduced by heating through the reduction in $E$, these postbuckling phenomena are very likely to be observed in beams in typical fires [10].

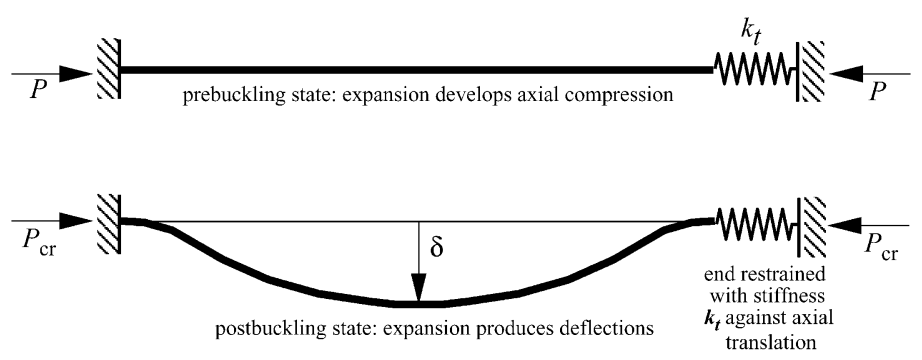

Fig. 8. Heating of beam with finite axial restraint. 


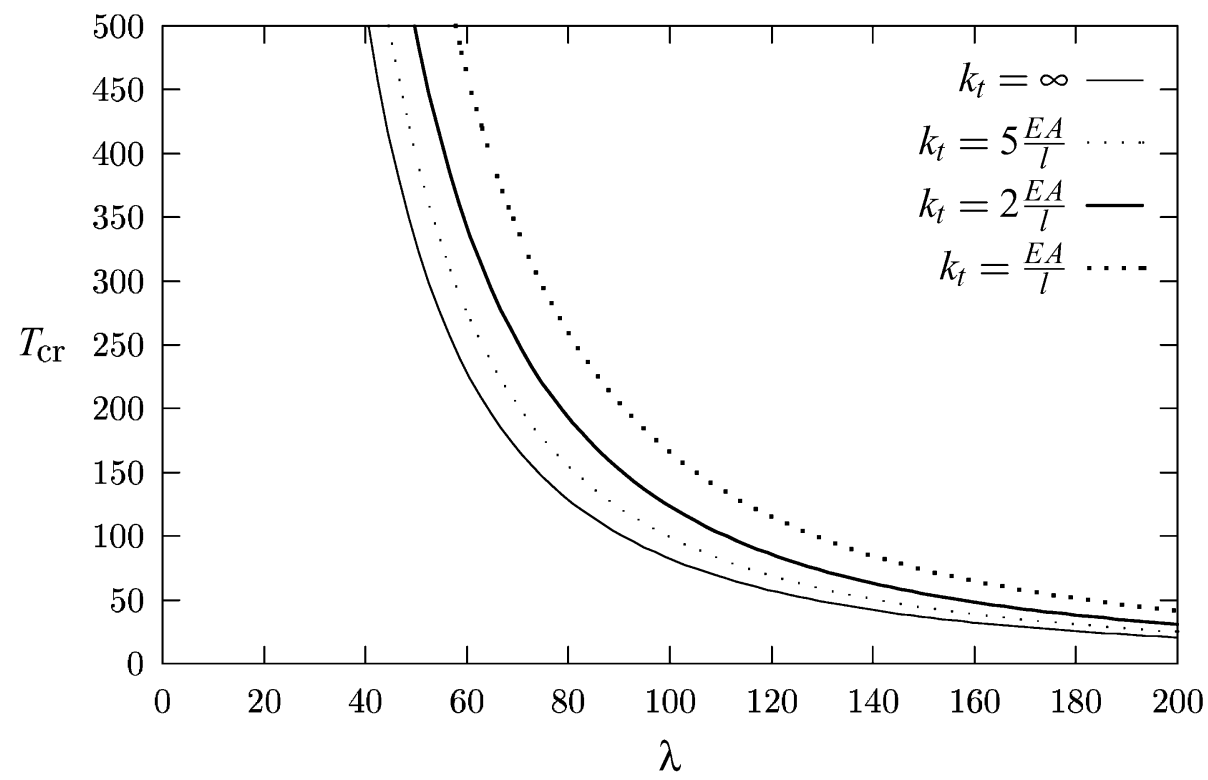

Fig. 9. Buckling temperatures for thermal expansion against finite lateral restraint.

\section{Thermal bowing}

In Section 2, we discussed the effects of uniform temperature rise on axially restrained beams. In real fires the temperature distributions are anything but uniform. In a small to moderate size compartment of a regular shape one may assume that the compartment temperature will be roughly uniform at a given ime. The temperature of the structural members in the compartment depends upon the material they are made of and other details of geometry, construction and design (such as insulation). Concrete beams and slabs on the ceiling of the compartment can be subjected to very high temperature gradients due to the very slow rates of heat transfer to concrete. Therefore, the surfaces exposed to fire will be at a much higher temperature than the surfaces on the outside of the compartment. This causes the inner surfaces to expand much more than the outer surfaces inducing bending in the member. This effect is called thermal bowing and is one of the main reasons of the deformations of concrete slabs and masonry walls in fires. Another very important source of thermal bowing in composite beams/slabs is the large difference between the temperatures of the steel joist and the slab. This effect is much more important in the early stages of the fire when steel retains most of its strength.

Relationships can be derived for thermal bowing analogous to the one derived earlier for thermal expansion. Fig. 10 shows a beam subjected to a uniform temperature gradient through its depth $(d)$ along its whole length $(l)$. Assuming the 


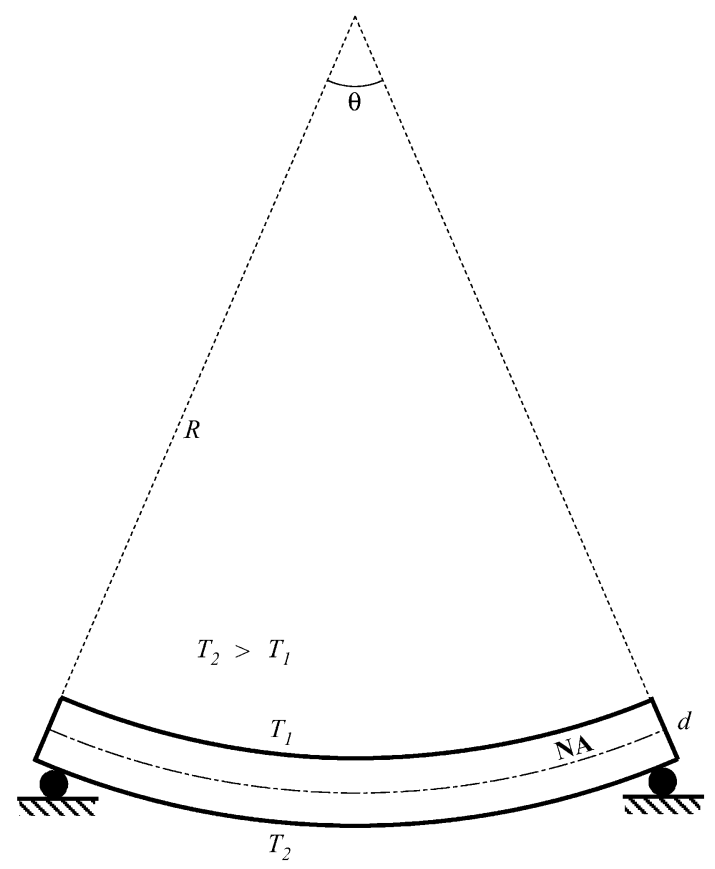

Fig. 10. Simply supported beam subjected to a uniform thermal gradient.

beam is simply supported (as shown in Fig. 10), we can derive the following relationships:

1. The thermal gradient $\left(T_{, \mathrm{y}}\right)$ over the depth is,

$$
T_{\mathrm{y}}=\frac{T_{2}-T_{1}}{d} .
$$

2. A uniform curvature $(\phi)$ is induced along the length as a result of the thermal gradient,

$$
\phi=\alpha T_{\text {, }}
$$

3. Due to the curvature of the beam, the horizontal distance between the ends of the beam will reduce. If this reduction is interpreted as a contraction strain (not literally) $\varepsilon_{\phi}$ (analogous to the thermal expansion strain $\varepsilon_{T}$, earlier), then the value of this strain can be calculated from analysing Fig. 10 as:

$$
\varepsilon_{\phi}=1-\frac{\sin (l \phi / 2)}{l \phi / 2} \text {. }
$$

Now consider the laterally restrained beam of Fig. 3. If a uniform thermal gradient $T_{\text {,y }}$ (without any average rise in temperature) is applied to this beam (as shown in Fig. 11), then the result is a thermally induced tension in the beam and corresponding reactions at the support (opposite to the pure thermal expansion case 


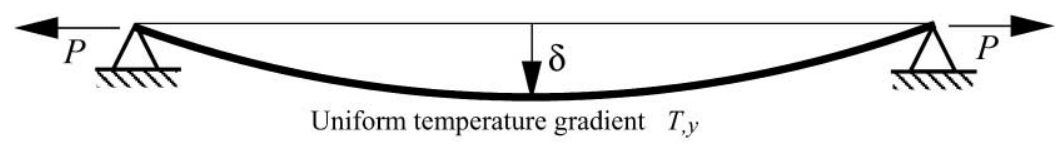

Fig. 11. Laterally restrained beam subjected to a uniform thermal gradient.

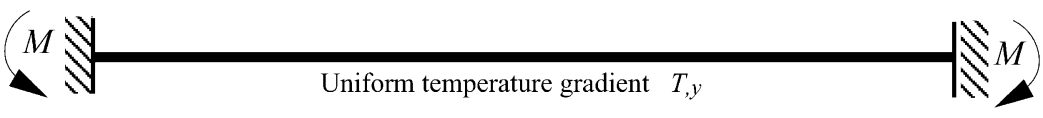

Fig. 12. Fixed end beam subjected to a uniform thermal gradient.

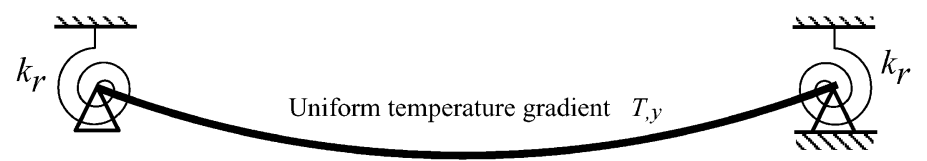

Fig. 13. Beam with finite rotational restraint with a uniform thermal gradient.

discussed earlier). This is clearly caused by the restraint to end translation against the contraction strain $\left(\varepsilon_{\phi}\right)$ induced by the thermal gradient.

Fig. 12 shows a fixed ended beam (by adding end rotational restraints to the beam of Fig. 11) subjected to a uniform temperature gradient through its depth. Recall that a uniform curvature $\phi=\alpha T_{\text {, }}$ exists in a simply supported beam subjected to gradient $T_{, \mathrm{y}}$. If that beam is rotationally restrained by support moments $M$ (uniform along length) an equal and opposite curvature induced by the support moments cancels out the thermal curvature and, therefore, the fixed ended beam remains 'straight' with a constant moment $M=E I \phi$ along its length.

From the above discussion it is clear that the effect of boundary restraints is crucial in determining the response of structural members to thermal actions. The key conclusion to be drawn from the discussion so far is that, thermal strains will be manifested as displacements if they are unrestrained or as stresses if they are restrained through counteracting mechanical strains generated by restraining forces.

As discussed earlier for lateral restraint, perfect rotational restraint is also not very easily achieved in real structures (other than for symmetric loading on members over continuous supports, without any hinges from strength degradation). Fig. 13 shows a beam restrained rotationally at the ends by rotational springs of stiffness $k_{\mathrm{r}}$. In this case, the restraining moment in the springs as a result of a uniform thermal gradient $T_{\text {,y }}$ can be found to be

$$
M_{k}=\frac{E I \alpha T_{, \mathrm{y}}}{\left(1+2 E I / k_{\mathrm{r}} l\right)} \text {. }
$$

This equation implies that if the rotational restraint stiffness is equal to the rotational stiffness of the beam $(E l / l)$ then the moment it attracts will be about a third of a fixed support moment. 


\section{Deflections}

In the previous sections, we have looked at the overall behaviour of beams subjected to expansion and bowing for various restraint conditions. One interesting aspect of structural response to fire is the large deflections that are found in structural members such as beams and slabs. Large deflections are normally associated with the loss of strength in structures under ambient conditions. In the case of fire, such a simple interpretation can be highly misleading. Both the thermal mechanisms discussed earlier (thermal expansion and thermal bowing) result in large deflections; however, the state of stress associated with a member subjected to varying degrees of these two mechanisms is not unique for a given deflection and a large range of stress states exist (large compression or tension or very low stresses) depending upon the temperature distribution in the member and its material properties and restraint conditions.

The chief reason for large deflections is that the structural member tries to accommodate the additional length generated by thermal expansion, given that it is not possible for it to expand longitudinally due to end restraints. Consider a slender beam (very low buckling temperature) subjected to uniform heating against rigid lateral restraints (as in Fig. 3). Buckling will occur very early (at very low elastic strains), after which any further expansion will make the beam deflect outwards. The resulting midspan deflection $\delta$ can be approximated quite accurately by

$$
\delta=\frac{2 l}{\pi} \sqrt{\varepsilon_{\mathrm{T}}+\frac{\varepsilon_{\mathrm{T}}^{2}}{2}},
$$

which is an approximation of the deflection of a sine curve of length $l\left(1+\varepsilon_{\mathrm{T}}\right)$, where $\varepsilon_{\mathrm{T}}$ is the thermal expansion strain $(\alpha \Delta T)$.

If the same beam is subjected to a uniform thermal gradient producing no net expansion, only bowing as in Fig. 11, the response is then determined by the flexuretension interaction. The tensile $P-\delta$ moments restrain the curvature imposed by the thermal gradients and limit deflections. The deflections result from the tensile strains produced in the beam, i.e.

$$
\varepsilon_{\mathrm{t}}=\frac{P}{E A}
$$

and the deflections can then be determined by

$$
\delta=\frac{2 l}{\pi} \sqrt{\varepsilon_{\mathrm{t}}+\frac{\varepsilon_{\mathrm{t}}^{2}}{2}} .
$$

The tensile force $P_{\mathrm{t}}$ can be determined by substituting Eq. (12) into Eq. (13) and solving a quadratic equation for $P_{\mathrm{t}}$,

$$
P_{\mathrm{t}}=\left(\sqrt{\frac{1}{2}\left(\frac{\pi \delta}{l}\right)^{2}+1}-1\right) E A .
$$


In order to determine the deflection $y(x)$ in the beam of Fig. 11 for a given curvature $\phi$ (arising from a thermal gradient), a differential equation solution can be written as follows:

For a simply supported beam subjected to a uniform curvature $\phi$ one can write

$$
\frac{\mathrm{d}^{2} y}{\mathrm{~d} x^{2}}=\phi
$$

If the beam is laterally restrained as in Fig. 11, then a tensile force $P$ will be generated causing a moment $P y$ over the length of the beam, therefore,

$$
\frac{\mathrm{d}^{2} y}{\mathrm{~d} x^{2}}=\phi+\frac{P y}{E I}
$$

or

$$
\frac{\mathrm{d}^{2} y}{\mathrm{~d} x^{2}}-k^{2} y=\phi
$$

where,

$$
k=\sqrt{\frac{P}{E I}} .
$$

The solution of this equation is,

$$
y(x)=-\frac{\phi}{k^{2}}\left(\frac{\cosh k l-1}{\sinh k l} \sinh k x-\cosh k x+1\right) .
$$

It may be seen that Eqs. (14) and (16) form a set of non-linear equations. These equations can be solved using an appropriate iterative technique (bisection, NewtonRaphson) to obtain the tensile forces and deflections for thermal gradient dominated problems.

\section{Combinations of thermal expansion and thermal bowing}

In the previous sections, the response of beams to either the thermal expansion or thermal bowing has been considered in isolation. To study the combined response, let us first consider the case of a fixed ended beam as shown in Fig. 14, which is both

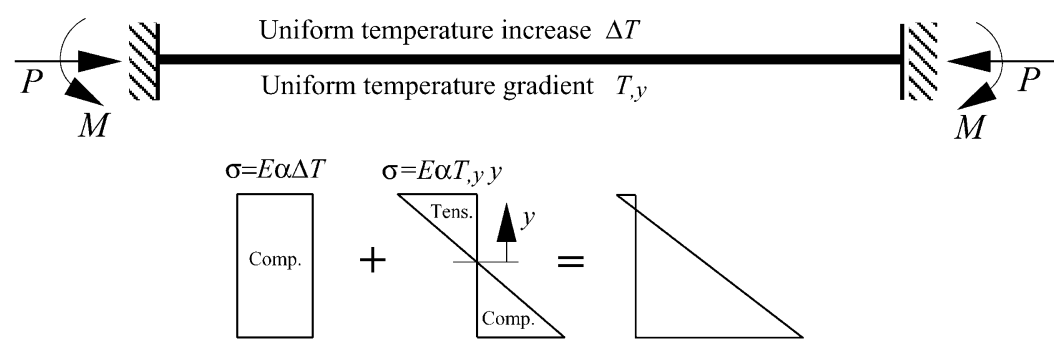

Fig. 14. Combined thermal expansion and bowing in a fixed ended beam. 
rotationally and translationally restrained at both ends. If this beam is subjected to a mean temperature rise and through a depth thermal gradient, then it will experience a uniform compressive stress because of restrained expansion and a uniform moment due to the thermal gradient. The stresses on any typical cross-section because of the combined effect of the two thermal actions are also shown in Fig. 14. It is clear that the bottom of the beam will experience very high compressive stresses, while the top may be anywhere between significant compression to significant tension.

The above scenario is a common one in composite frame structures such as Cardington. The composite action of a steel joist, framing into an interior column, with a continuous slab over it, produces conditions similar to a fully fixed support (as in Fig. 14). The high compressions resulting from the combined effect of thermal actions as described above almost invariably produce local buckling in the lower flange of the steel joist very early in a fire. This is why local buckling of the lower flanges is such a common occurrence in fires, as seen in all Cardington tests [11] and other fires [5].

Once local buckling has occurred, the pattern of stresses at the ends of the composite beam changes. The hogging moment is relieved by the hinge produced by local buckling and the end restraint conditions change to the one shown in Fig. 11 . As this happens quite early in real fires, the end conditions described by Fig. 11 are those that govern the behaviour of a composite beam for most of the duration of the fire.

\subsection{Combined thermal expansion and bowing in laterally restrained beams}

The fundamental pattern of behaviour of a beam whose ends are laterally restrained (but rotationally unrestrained, see Fig. 11), subjected to thermal expansion and thermal bowing separately was established in the previous sections. Restrained expansion resulted in compression and bowing resulted in tension. This helped to illustrate that two opposite stress regimes can occur depending upon the thermal regime applied; however, the apparent response of the beam is the same (i.e. downward deflection). The main parameters that determine the response are an average temperature equivalent rise $(\Delta T)$ and an average equivalent thermal gradient $\left(T_{, y}\right)$. For a practical application of the expressions presented in this paper, these parameters must be determined first. A procedure for determining these values in beams of any general cross-section with any temperature distribution over its depth is given in Ref. [12].

To study the effects of the applying combinations of thermal expansion and thermal bowing, we define the effective strain as follows:

$$
\varepsilon_{\mathrm{eff}}=\varepsilon_{\mathrm{T}}-\varepsilon_{\phi}
$$

The variation of $\varepsilon_{\mathrm{eff}}$ (for various thermal regimes) can produce a large variety of responses. Positive values of $\varepsilon_{\text {eff }}$ imply compression (or the effect of mean temperature rise is dominant) and negative values imply tension (or the effect of thermal gradients is dominant). Fig. 15 shows the variation of $\varepsilon_{\mathrm{eff}}$ for different values of 


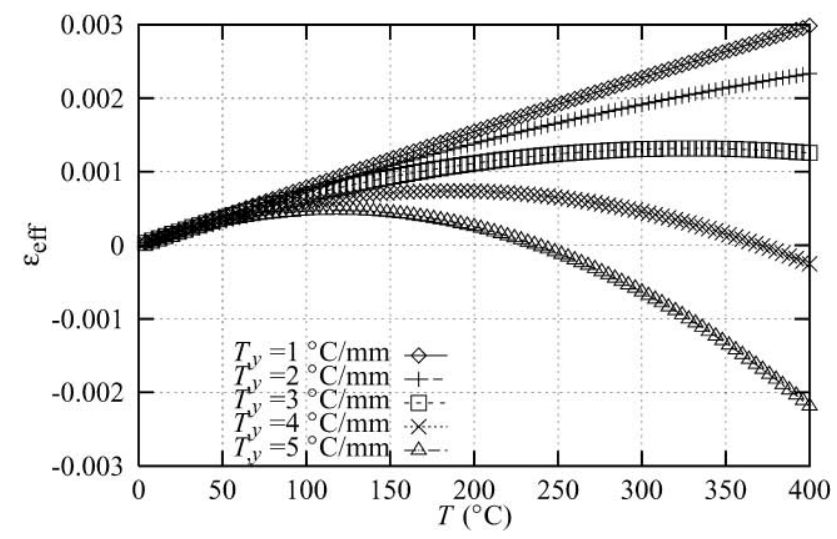

Fig. 15. Effective expansion strains.

Step 1: Impose a temperature rise $\Delta \mathrm{T}$

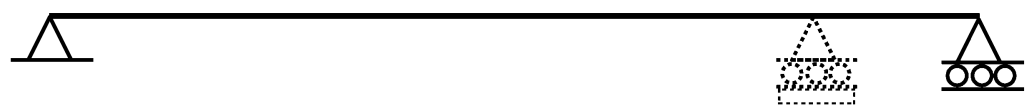

Step 2 : Impose a curvature $\alpha \mathrm{T}$,y to return support to the original position

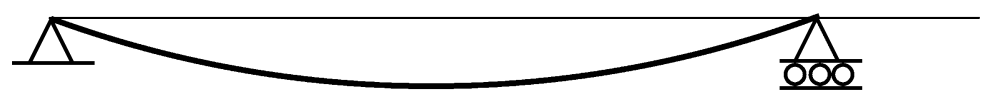

Fig. 16. Case 1: zero stress.

thermal gradient when the temperature is increased from 0 to $400^{\circ} \mathrm{C}$ ( $\varepsilon_{\mathrm{eff}}$ is plotted for a linear increase in gradient against temperature).

Case 1: Zero stress in the beam $\left(\varepsilon_{\mathrm{eff}}=0\right)$

Fig. 16 shows an interesting theoretical case. If the implied combination of $\varepsilon_{\phi}$ and $\varepsilon_{\mathrm{T}}$ are applied, then

- There will be no stresses in the beam. All thermal strains are converted into displacement as seen in the figure.

- The deflection of the beam will be entirely due to thermal bowing to accommodate the excess length generated by expansion.

- The deflection response of this beam can be analytically expressed as the increase in the length of the elastic curve of the beam versus its deflection. Fig. 17 shows the number of length increases vs. midspan deflection plots based on assumed curve shapes. The figure shows that the shape of the curve chosen does not matter much, therefore, the formula given earlier based upon the sine curve (Eq. (11)) can 


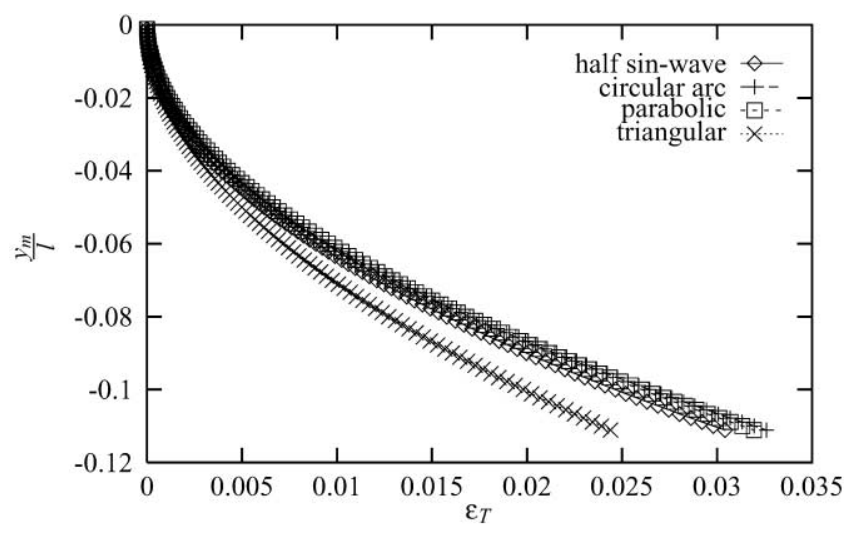

Fig. 17. Strain vs. non-dimensional midspan deflection (per unit length).

be used to get a good approximation of the midspan deflection $y_{\mathrm{m}}$, i.e.

$$
y_{\mathrm{m}}=\frac{2 l}{\pi} \sqrt{\varepsilon_{\mathrm{T}}+\frac{\varepsilon_{\mathrm{T}}^{2}}{2}} .
$$

Case 2: Thermal expansion dominant $\left(\varepsilon_{\mathrm{eff}}>0\right)$

If $\varepsilon_{\mathrm{T}} \gg \varepsilon_{\phi}$, then thermal expansion dominates and a two-stage response is produced consisting of pre- and post-buckling phases. The thermal expansion produced is partly used up in generating mechanical strains and partly in generating deflections. This is governed by the magnitude of $\varepsilon_{\mathrm{eff}}$ which is the component that generates stresses to progress the beam towards buckling. The $\varepsilon_{\phi}$ component annihilates a part of the expansion and produces deflections by imposing curvature with the available excess length. The pre-buckling deflections $\left(y_{\mathrm{m}}^{-}\right)$will, for a small part, result from the elastic bending of the beam, while a larger part will generally come from the deflection resulting from the imposed curvature $\left(y_{\mathrm{m}}(\phi)\right)$.

$$
y_{\mathrm{m}}^{-}=\frac{y_{0}}{1-\left(\Delta T / \Delta T_{\mathrm{cr}}\right)}+y_{\mathrm{m}}(\phi)
$$

Here, $y_{0}$ can be interpreted as the initial elastic deflection before the fire because of the imposed loads on the beam and $y_{\mathrm{m}}(\phi)$ is the extra deflection due to thermal bowing given by

$$
y_{\mathrm{m}}(\phi)=\frac{2 l}{\pi} \sqrt{\varepsilon_{\phi}+\frac{\varepsilon_{\phi}^{2}}{2}} .
$$

The presence of the gradient clearly delays the buckling event and the critical buckling temperature $\left(\Delta T_{\mathrm{cr}}\right)$ is increased to,

$$
\Delta T_{\mathrm{cr}}=\frac{1}{\alpha}\left(\frac{\pi^{2}}{\lambda^{2}}+\varepsilon_{\phi}\right) .
$$




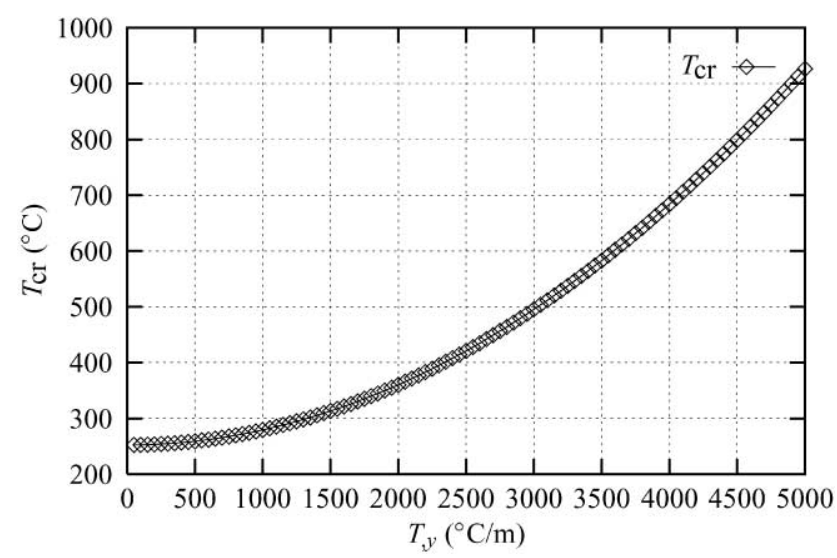

Fig. 18. Critical buckling temperatures vs. thermal gradient.

Fig. 18 shows the typical variation in buckling temperature with the change in gradient (for a beam of slenderness ratio $l / r$ equal to 70 ).

The post-buckling deflections will keep increasing because of all the further expansion strains $\varepsilon_{\mathrm{T}}^{+}$as in Eq. (11), i.e.

$$
y_{\mathrm{m}}^{+}=\frac{2 l}{\pi} \sqrt{\varepsilon_{\mathrm{T}}^{+}+\frac{\varepsilon_{\mathrm{T}}^{+2}}{2}} .
$$

The thermal bowing deflection added to the elastic deflections (due to $P-\delta$ moments and loading) will again act as 'imperfections' to 'straightness' of the beam and produce a smooth variation of beam midspan deflection with temperature until the large displacement post-buckling mode begins (identified by the change of curvature of the temperature deflection curve). This also has the effect of reducing the development of compression forces in the beam (the beam displaces more for lower compressions because of the additional bowing displacements increasing the $P-\delta$ moments).

Case 3: Thermal bowing dominant $\left(\varepsilon_{\mathrm{eff}}<0\right)$

When $\varepsilon_{\phi} \gg \varepsilon_{\mathrm{T}}$, the deflection response will be the sum of the two components,

1. Deflection caused by bowing of the excess length generated through expansion as before, i.e.

$$
\left(y_{\mathrm{m}}\right)_{1}=\frac{2 l}{\pi} \sqrt{\varepsilon_{\mathrm{T}}+\frac{\varepsilon_{\mathrm{T}}^{2}}{2}} .
$$

2. The tensile strain $\varepsilon_{\mathrm{t}}$ produced by the tension $\left(P_{\text {eff }}\right)$ caused by the excess contraction strain $\left(\varepsilon_{\mathrm{eff}}\right)$ as in the case of pure thermal gradients

$$
\varepsilon_{\mathrm{t}}=\frac{P_{\mathrm{eff}}}{E A}
$$




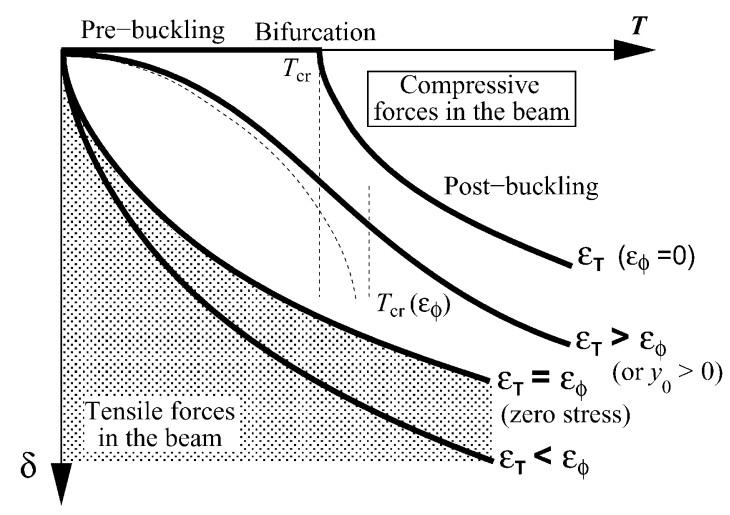

Fig. 19. Temperature deflection responses for combinations of $\varepsilon_{\mathrm{T}}$ and $\varepsilon_{\phi}$.

which will produce further deflections as

$$
\left(y_{\mathrm{m}}\right)_{2}=\frac{2 l}{\pi} \sqrt{\varepsilon_{\mathrm{t}}+\frac{\varepsilon_{\mathrm{t}}^{2}}{2}} .
$$

The tension $P_{\text {eff }}$ and the deflections can then be determined according to the iterative procedure suggested in the previous section on deflections.

Finally, Fig. 19 shows the main types of deflection responses that may be observed if a laterally restrained beam is exposed to the combinations of thermal actions discussed above.

\section{Criterion for the various types of responses}

From the discussion above, a simple criterion for all the various types of responses observed can be developed. If $\varepsilon_{\text {eff }}$ is similar to $\pi^{2} / \lambda^{2}$, then there will be no buckling as not enough compression is generated. A dimensionless number $\zeta$ may be defined as follows to categorise the various responses

$$
\zeta=\frac{\varepsilon_{\mathrm{T}}-\varepsilon_{\phi}}{\pi^{2} / \lambda^{2}}
$$

To summarise,

1. $\zeta \gg 1$ typically generates pre- and post-buckling type deflection responses with thermal expansion and dominant compression. The compression force patterns are as discussed earlier in the restrained thermal expansion section.

2. $\zeta \simeq 1$ generates responses where most of the thermal expansion is converted into deflection, but there are negligible stresses in the beam (close to the zero stress case discussed earlier). 
3. $\zeta \ll 1$ generates thermal bowing dominated response with deflection patterns similar to the zero stress case and with considerable tensile forces in the beam which grow with the increase in the gradient.

In the above analysis, the effect of the relative axial and flexural stiffnesses of the beam has not been explicitly considered, such as an I-beam with a high radius of gyration $(r)$ and a slab with a much lower $r$. The actual midspan deflection in a laterally restrained beam subjected to both thermal expansion and thermal bowing is highly dependent upon the relative values of axial and flexural stiffnesses of the beam. A slender beam (of low flexural stiffness - such as the slab in isolation as considered here) does not have sufficient flexural stiffness to overcome the tensile axial stiffness and develop curvature (or 'bow') as much as a stocky beam (with relatively large flexural stiffness) would. Therefore, bowing deflection will be much lower in slender beams (as here) than in stocky composite beams. In the extreme situation, the actual deflection in a very slender beam can be slightly 'lower' with a combined thermal expansion and thermal bowing than the deflection caused only by thermal expansion (this is only when bowing is dominant-so the beam is in overall tension). This is caused in turn by the change in slope caused by bowing induced tensile forces at the ends introducing double-curvature in the beam (for a fixed-ended case).

\section{Numerical analysis of the simple beam model}

The analytical approach developed above in order to fully understand the structural response to the heating regime has been checked numerically by modelling the same simple beam examples in ABAQUS. The data for the analysed beam were as follows:

- length $(l)=9000 \mathrm{~mm}$,

- modulus of elasticity $(E)=210,000 \mathrm{~N} / \mathrm{mm}^{2}$,

- coefficient of thermal expansion $(\alpha)=8 \times 10^{-6}$,

- area $A=5160 \mathrm{~mm}^{2}$,

- second moment of area $I=8.55 \times 10^{7} \mathrm{~mm}^{2}$.

Therefore, the slenderness ratio $l / r$ of the beam is approximately equal to 70 . This calculation is limited to the investigated simple beam model restrained laterally, but free to rotate at its ends as in Fig. 11. The results confirm the theoretical solutions derived for the response of the beam to thermal bowing and thermal expansion. Fig. 20 shows the results of the numerical analysis in terms of the deflections and axial forces produced when the beam is subjected to a mean temperature rise (uniform over the length) of $400^{\circ} \mathrm{C}$ and an effective thermal gradient through the depth of the beam. The temperature increase, $\Delta T$ and thermal gradient, $T_{\text {,y }}$ were applied to the simple numerical beam model at a constant rate from zero to their maximum values. The deflection as a result of pure restrained thermal expansion shows the double curvature shape of the pre-/post-buckling responses (see Fig. 20). When a gradient is also applied to the model and the response of the beam is 

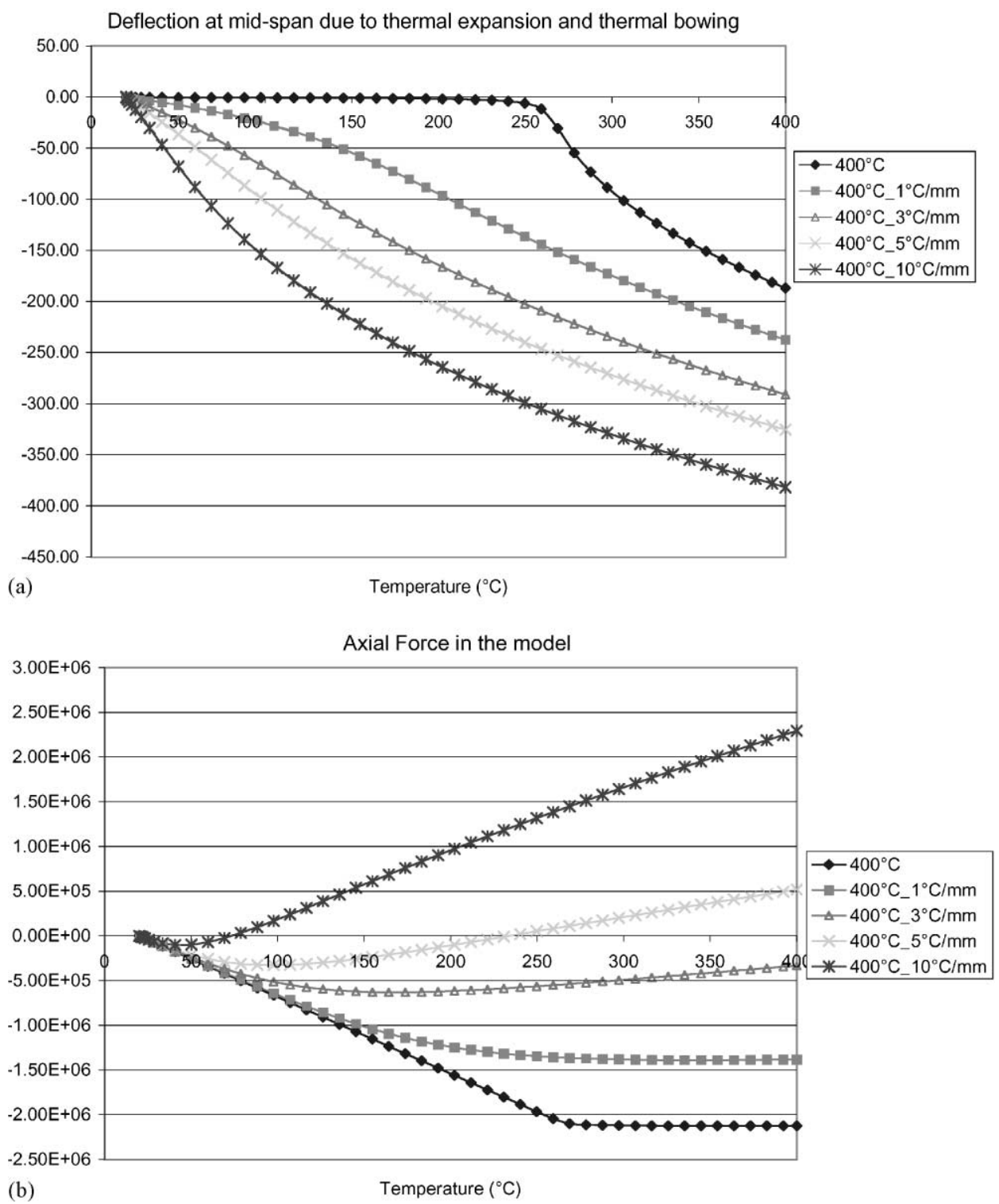

Fig. 20. Numerical model results for combined thermal expansion and thermal bowing: (a) deflections, and (b) axial forces.

governed by the interaction between thermal bowing and restrained thermal expansion the deflected shape becomes smoother, indeed for a specific combination of mean temperature rise and temperature gradient the response will be similar to linear. At large gradients $\left(T_{\mathrm{y},}=10^{\circ} \mathrm{C} / \mathrm{mm}\right)$, when the response is dominated by thermal bowing the deflected shape is very non-linear. 
The corresponding axial forces are also plotted in Fig. 20. When a mean temperature rise of $400^{\circ} \mathrm{C}$ alone is applied to the model and the response of the beam is governed purely by restrained thermal expansion, the axial force is in high compression. When the model is subjected to a combination of mean temperature rise and temperature gradient, the axial force becomes smaller in compression and at high gradients moves into tension. The axial force at the beginning of the analysis is always in compression because the mean temperature and the gradient are applied to the model linearly from zero to their maximum values and are governed by the development of the effective strains, $\varepsilon_{\mathrm{eff}}$, as shown in Fig. 15.

The actual values of deflections and forces in the numerical exercise above can be estimated using the formulas given here. For instance, for the case of a temperature rise of $400^{\circ} \mathrm{C}$ the compression force is simply the Euler buckling load $\left(\pi^{2} E A / \lambda^{2}\right)$ equal to $2170 \mathrm{kN}$ (approx.). The deflection for this case can be obtained from subtracting the elastic compression strain $\left(\pi^{2} / \lambda^{2}\right)$ from the thermal strain $\left(\varepsilon_{\mathrm{T}}\right)$ to obtain the strain that produces the deflections, therefore,

$$
y_{\mathrm{m}} \simeq \frac{2 l}{\pi} \sqrt{\varepsilon_{\mathrm{T}}-\frac{\pi^{2}}{\lambda^{2}}}
$$

which produces a value of approximately $200 \mathrm{~mm}$ (within 10\%) of the numerical calculation above. The difference is because the numerical calculation is fully geometrically non-linear while the above formulas are based on first order definitions of strain.

If all the thermal strains were to produce deflection (by appropriate combination of $\varepsilon_{\mathrm{T}}$ and $\varepsilon_{\phi}$ ) then the internal forces would be very low and the deflection would be approximately $324 \mathrm{~mm}$ (from Eq. (11)) which lies between the cases of $T_{, \mathrm{y}}=$ $3-5^{\circ} \mathrm{C} / \mathrm{mm}$. It may be noted from Fig. 20 that the axial force in the beam moves from compression to tension between these values (suggesting that for the deflections in the region of $324 \mathrm{~mm}$ ) the forces in the beam will be insignificant.

The tensile forces and deflections for large gradients can be calculated from iteratively solving the set of non-linear Eqs. (14) and (16).

The above analysis clearly highlights the large range of deflected shapes and axial forces that are possible as a result of the interaction between thermal expansion and thermal bowing.

\section{Other important factors}

The discussion above has focussed upon the effects of thermal expansion and thermal bowing and illustrated the large variety of responses possible in real composite frame structures. Analytical expressions have been presented which allow a good quantitative estimate of forces and deflections to be made for simple structures.

Effects of thermal degradation and imposed loading were found to be relatively less important in the modelling of Cardington Tests [13,14]. The effect of strength 
degradation was shown to change the development of compressive forces in a restrained beam in Figs. 6 and 7. The loading on a beam in a large displacement configuration (through thermal effects) will be carried very effectively in a catenary (or tensile membrane) behaviour. It is clear from the above discussion that for the most likely combinations of thermal actions $\left(\varepsilon_{\mathrm{T}}\right.$ and $\left.\varepsilon_{\phi}\right)$ the mechanical strains in a member are likely to be very low (compression or tension). If this is true and that the thermal degradation has been contained in the surface layers, then the tensile strains induced by membrane mechanisms should be carried quite reliably. This is, however, an area which needs further extensive investigation using all the standard research techniques, experimental, computational and theoretical analyses.

Restraint conditions can certainly have a major effect on the distribution of the internal forces and the displacements that occur as has been illustrated by the simple theoretical and computational analyses in this paper. The degree of restraint available also changes during a fire, for instance the rotational restraint available to the composite beam at the beginning is lost quite early (around $200^{\circ} \mathrm{C}$ ) [10] due to the local buckling of the steel beam and tensile capacity of the slab being reached. Rotational restraints result in increasing hogging moments until a 'plastic hinge' is achieved. Lateral translation restraints produce compression forces if thermal expansion is dominant and tension forces if thermal bowing is dominant. The amount of restraint required is not large to produce buckling as floor structures are usually very slender. The source of this restraint is obvious for interior compartments - the colder and stiffer surrounding structure. For exterior compartments, it is not so clear if sufficient restraints are still available. It is likely that sufficient restraint to lateral expansion is available at exterior boundaries through the actions of tension rings [3]. At large deflections, lateral restraints provide an anchor to the tensile membrane mechanisms. Again, it is likely that sufficient lateral restraint is available at exterior boundaries through the action of compression rings [8]. This, however, is a matter of much greater importance than the restraint to thermal expansion as the survival of the floor system ultimately depends upon the reliability of the tensile membrane mechanism. This again is a key question for further investigation. An yield analysis based estimation of ultimate capacity [15] may be useful in the absence of better models, however, it is likely to be conservative in the case of structures in fire, where the curved deformed 'shape' of the floor slabs (suitable for effective tensile membrane mechanism to develop) along with low tensile strains may produce large capacity. This, however, needs to be investigated further and until then conservatism is certainly the preferable option.

Another very important factor that has not been investigated here is the effect of the compartment geometry. This can have a large effect on the development of thermally induced forces and deflections in the heated structural members. The principle that allows one to make a quantitative assessment of the effect of compartment geometry, is compatibility. For instance, for a rectangular fire compartment, the thermal expansion in the shorter direction will be smaller than the expansion in the longer direction. This can lead to an increase in compression in the longer direction (because compatibility does not allow it to deflect as much as its thermal expansion demands). In the shorter one, the reverse happens, compatibility forces 
the deflections in this direction to be somewhat larger than thermal expansion would allow resulting in lower compressions or even tensile forces. This has been identified clearly in the modelling of the British Steel restrained beam test $(3 \mathrm{~m} \times 8 \mathrm{~m})$, where the midspan ribs are in tension. This allows the redistribution of the thermally induced forces. See Ref. [12] for further information on this topic.

\section{Conclusions}

It is now well recognised that contrary to popular belief, composite steel framed structures possess a much larger inherent fire resistance than that apparent from testing single steel members in fire furnaces. It is also accepted that the current prescriptive approaches of designing such structures are overly conservative and not based on rational principles. It is therefore possible to construct these strcutures much more economically, without any loss of fire resistance, by removing or drastically reducing the fire protection of steel members. However, to fully exploit the considerable reserves of strength, it is imperitive that the mechanics of whole steel frame structure behaviour in fire is understood well. The ideas presented in this paper are a step in this direction.

The fundamental principles presented in this paper provide a means of estimating forces and displacements in real structures with appropriate idealisations. Such estimates can be of considerable use in assessing the results from more rigorous numerical analyses or they can be used in design calculations. There are, however, a considerable number of very important issues that remain to be investigated as mentioned in the previous section. Considerable effort is required to address these issues to satisfaction before a complete set of principles can be developed. Ref. [12] describes an early attempt at using these principles to model a real fire test with encouraging results and demonstrates the potential of such analyses. Further work is being carried out to put the ideas presented here to more rigorous testing.

\section{References}

[1] Final Report of the DETR-PIT Project. Behaviour of steel framed structures under fire conditions. Technical report, University of Edinburgh, 2000.

[2] Bravery PNR. Cardington large building test facility, construction details for the first building.Technical report, British Steel plc (now CORUS), 1993 [Internal report].

[3] Rotter JM, Sanad AM, Usmani AS, Gillie M. Structural performance of redundant structures under local fires. Proceedings of the Interflam'99, Eighth International Fire Science and Engineering Conference, Edinburgh, Scotland, 29 June-1 July 1999. p. 1069-80.

[4] Rotter JM, Usmani AS. Fundamental principles of structural behaviour under thermal effects. Proceedings of the First International Workshop on Structures in Fire, Copenhagen, Denmark, June 2000.

[5] S.C.I. Investigation of Broadgate Phase 8 Fire. Structural fire engineering. Steel Construction Institute, Ascot, Berkshire, 1991.

[6] O'Connor DJ, McAllister B, Munro J, Bennett HR. Determination of the fire endurance of model concrete slabs using a plastic analysis methodology. Struct Eng 1995;73(19):325-30. 
[7] Franssen JM, Schleich JB, Cajot LG, Azpiazu W. A simple model for the fire resistance of axially loaded members - comparison with experimental results. J Construct Steel Res 1996;37:175-204.

[8] Wang YC. Tensile membrane action in slabs and its application to the Cardington fire tests. Technical report, Paper presented to the second Cardington Conference, Building Research Establishment, 12-14 March 1996.

[9] Huang Z, Burgess IW, Plank RJ. Three-dimensional modelling of two full-scale fire tests on a composite building. Proc Inst Civil Eng Struct Build 1999;134:243-55.

[10] Sanad AM, Rotter JM, Usmani AS, O'Connor M. Composite beam in buildings under fire. Fire Safety J 2000;35:165-88.

[11] Swinden Technology Centre British Steel plc. The behaviour of multi-storey steel framed buildings in fire. Technical report, European Joint Research Program Report. British Steel plc (now CORUS), 1999.

[12] Usmani AS. Application of fundamental structural mechanics principles in assessing the Cardington fire tests. Proceedings of the First International Workshop on Structures in Fire, Copenhagen, Denmark, June 2000.

[13] Sanad AM. Effect of increasing live loads on bs/test1. Technical report PIT/SM1, School of Civil and Environmental Engineering, University of Edinburgh, 2000. Submitted to DETR(UK) as part of the final report for the PIT project Behaviour of steel frame structures under fire conditions.

[14] Sanad AM. Effect of increasing steel section in the bs/test1 composite beam. Technical report PIT/ SM2, School of Civil and Environmental Engineering, University of Edinburgh, 2000. Submitted to DETR(UK) as part of the final report for the PIT project Behaviour of steel frame structures under fire conditions.

[15] Bailey CG, Moore DB. The structural behaviour of steel frames with composite floorslabs subject to fire: part 1: theory, part 2: design. Struct Eng 2000;78:19-33. 